\title{
Global and Seasonal Scintillation Morphology in the Equatorial Region Derived from ROCSAT-1 In-situ Data
}

\author{
Yen-Hung $\mathrm{Liu}^{1, *}$, Chao-Han Liu ${ }^{1,2}$, and Shin-Yi Su${ }^{3}$ \\ ${ }^{1}$ Department of Electrical Engineering, National Central University, Jhongli, Taiwan \\ ${ }^{2}$ Academia Sinica, Taipei, Taiwan \\ ${ }^{3}$ Institute of Space Science, and Center for Space and Remote Sensing Research, National Central University, Jhongli, Taiwan
}

Received 3 March 2011, accepted 30 June 2011

\begin{abstract}
The global/seasonal distributions of the scintillation occurrence rate are obtained from the in-situ density measurement of the ROCSAT-1 using a modified procedure reported by Wernik et al. (2007). A least-squares curve fitting in the optimal trust region is used to obtain the spectral slope for the density irregularity structure and the outer scale of the scintillation. The distribution of the $S_{4}$ index for the weak scintillation $\left(S_{4}<0.3\right)$ is almost identical to that of the equatorial irregularity distribution reported in the literature. However, as the scintillation becomes stronger $\left(0.3<S_{4}<0.6\right)$, the latitudinal distribution moves to the equatorial ionization anomaly (EIA) region. In addition, the distributions of the outer scale values that are useful for the study of the physical evolution of the irregularity structure are also obtained. The occurrence distribution of scintillation activity with several parameters such as dip-latitude, longitude, local time, solar activity, and geomagnetic activity during different seasons are presented and discussed in this paper.
\end{abstract}

Key words: Equatorial irregularities, Scintillations, Outer scale and $\mathrm{S}_{4}$-index

Citation: Liu, Y. H., C. H. Liu, and S. Y. Su, 2012: Global and seasonal scintillation morphology in the equatorial region derived from ROCSAT-1 in-situ data. Terr. Atmos. Ocean. Sci., 23, 95-106, doi: 10.3319/TAO.2011.06.30.01(AA)

\section{INTRODUCTION}

The prior knowledge of current ionospheric conditions and the probability of scintillation occurrences are very important for various practical applications in communication and navigation systems at low latitude regions where scintillation is severe. If the probability of an occurrence and intensity rate is made available, then a system designer can have a reference to evaluate and design a system with a proper propagating channel passing through the ionosphere.

Scintillation morphology describing the scintillation occurrence rate at different geo-locations can be used for practical applications and understanding the physics of the development of irregularities. There are some studies which summarized the variation of scintillation occurrence rate from the morphology derived from the ground observations in the past. Aarons (1982) illustrated variations of the global scintillation phenomenon. In equatorial scintillation, the

\footnotetext{
* Corresponding author

E-mail: yhliu168@gmail.com
}

characteristics of irregularities were shown and variations of scintillation occurrence rates with longitude, magnetic activity and sunspot cycle were also presented. Basu and Basu (1985) summarized various hypotheses and physics of developing scintillation occurrence rates correlated to latitude and longitude in the equatorial region. In addition, the structure of the irregularity that causes scintillation and the modeling results were illustrated. Tsunoda (1985) used observations of equatorial scintillation at sunset at different locations to derive the hypothesis that the occurrence of scintillation peaks when the solar terminator is nearly aligned with the magnetic flux at a given longitude. In Basu et al. (1988), the mechanism causing variations in scintillation occurrence rates with solar activity was reported from the construction of scintillation morphology during solar maximum and minimum periods according to signal fade on the ground. In addition, the hypotheses in developing the longitudinal variation of scintillation occurrence rate around the equatorial region were discussed in Aarons (1993). 
There are empirical scintillation models which have been developed in coordination with global scintillation observations (Aarons 1982). Currently, Wideband ionospheric scintillation model (WBMOD) is the most popular climatological scintillation model which is divided into two groups, the propagation model and the environment models. The propagation model is a phase screen model provided by Rino (1979) and environment models describe the spatial distribution of parameters required by the propagation model and their temporal variations from an analysis of scintillation data and other data sets. The output of WBMOD provides a global prediction of scintillation activity as a function of radio frequency, day of year, time of day, geographic latitude and longitude, sunspot number and magnetic activity (Aarons 1982; Secan et al. 1995, 1997; Wernik et al. 2003). In $\mathrm{Li}$ and Liu (2004), the value of the scintillation index from WBMOD is used to evaluate the effect of ionospheric scintillation on the bit error rate performance for a specific system. However, there are some studies that report some disagreement with the prediction of scintillation activity in some regions (Cervera et al. 2001).

Therefore, this study is intended to refine the construction of global/seasonal/local-time scintillation occurrence distributions at low latitudes from in-situ satellite measurement data. In the past, there were case studies on the modeling of scintillation occurrences. Basu et al. (1976) noted that the OGO-6 ion density measurement and power law phase screen model with some assumed geophysical parameters are used to derive the occurrence rate of the VHF scintillation in winter. Wernik et al. (2007) provided a scintillation climatological model using the density observations from the DE2 satellite to derive the Northern Hemisphere high latitude scintillation distribution by a closed form expression of the phase screen theory provided by Rino (1979) with the ionospheric thickness and height derived from the IRI-95 model. In the present study, we use ion density measurements from the ROCSAT-1 satellite operating during high solar activity periods around the equatorial region to derive the scintillation distribution which can complement the model proposed by Wernik et al. (2007).

Because of a close relationship between an irregularity and scintillation (Wernik et al. 2003,2007), we can obtain a scintillation occurrence rate from irregularity structures. For the radiowave frequency in the $\mathrm{L}$ band communication and GPS systems, we can safely assume that the wave has weak refraction in the ionospheric layer and only the phase is distorted. Therefore, the phase screen theory is adopted to obtain the scintillation index $\mathrm{S}_{4}$ from the in-situ measurement of ion density from the ROCSAT-1. In addition, we have used a modified approach to obtain the spectral index of the density irregularity structure that is slightly different from what was reported in Wernik et al. (2007). In this modified approach, we are able to obtain the outer scale from each fitting of the irregularity structure. Thus the distribution of the outer scale related to the scintillation is also included in the current study.

The objective of this paper is to apply the optimum method, a least-squares curve fitting in the trust region, to derive the spectral index and outer scale to calculate the scintillation index from the phase screen model. The statistical distribution of the outer scale from the in-situ measurement data can be used to compare with many values presented in previous reports (Basu et al. 1976; Yeh and Liu 1977; Rino and Owen 1984)

\section{THE SCINTILLATION THEORY MODEL}

Under the conditions of forward scattering and Fresnel approximation, phase screen theory assumed that the ionosphere is a thin phase changing screen which modifies only the phase of the radio wave traversing through the ionospheric layer (Yeh and Liu 1982; Bhattacharyya et al. 1992). Therefore, it establishes a relationship between the spatial variation of the ionospheric electron density and the scintillation received on the ground.

Rino (1979) presents a close form expression for the scintillation index from one-dimensional spectral density function (SDF) of the in-situ measured electron density that is given by Wernik (2007)

$$
P_{s}\left(f_{i}\right)=\frac{C_{s} \Gamma\left(\frac{p}{2}\right)}{4 \pi^{2} \Gamma\left(\frac{p}{2}+1\right)} \cdot \frac{1}{V_{p}\left[q_{0}^{2}+\left(\frac{2 \pi f_{i}}{V_{p}}\right)^{2}\right]^{p / 2}}
$$

where $p$ is the one-dimensional spectral index, $q_{0}=2 \pi / \lambda_{0}$ is the outer scale wave number and $\lambda_{0}$ is the outer scale. $V_{p}$ is the effective probe velocity derived from $V_{p}^{2}=\vec{V}^{T} C \vec{V}$ where $\vec{V}$ is the true velocity of the probe in the reference coordinate system. $C$ is a matrix of irregularity elongation parameters, magnetic dip angle and inclination angle of the transverse irregularity axis (Rino and Fremouw 1977). The turbulence strength parameter at the satellite height, $C_{s}$, is given by the following equation (Rino 1979; Wernik 2007),

$C_{s}=8 \pi^{3 / 2}\left\langle\Delta N_{e}^{2}\right\rangle q_{0}^{p-1} \cdot \frac{\Gamma\left(\frac{p}{2}+1\right)}{\Gamma\left(\frac{p}{2}-\frac{1}{2}\right)} \quad, \quad p>1$

where $\left\langle\Delta N_{e}^{2}\right\rangle$ is the variance of electron density acquired by the probe onboard the satellite. The scintillation index $\mathrm{S}_{4}$ is then obtained from

$S_{4}^{2}=\left(r_{e} \lambda\right)^{2} L \sec \theta C_{s r} Z^{p / 2} \frac{\Gamma(1-p / 4)}{\pi^{1 / 2} \Gamma(p / 4+0.5) p} F$ 
where $r_{e}$ is the radius of the electron, $\lambda$ is the wavelength of the signal propagating through the ionosphere, $\theta$ is the zenith angle, and $Z$ is the Fresnel zone parameter which is described by the following equation

$Z=\frac{\lambda z_{R} \sec \theta}{4 \pi}$

where $z_{R}=z z_{s} /\left(z+z_{s}\right)$ is the effective distance in which $z_{S}$ is the distance to the satellite and $z$ is the distance to the phase screen. The Fresnel filtering factor $F$ in Eq. (3) depends on the anisotropy of irregularities, which is a function of magnetic local time and geomagnetic latitude (Rino 1979; Wernik et al. 2007).

As suggested by Wernik et al. (2007), the height of the phase screen, the irregularity thickness and the ratio of $C_{s}$ to $C_{s r}$, the turbulence strength parameter at the screen height, $C_{s r} / C_{s}=\left\langle\Delta N_{m}^{2}\right\rangle /\left\langle\Delta N_{s}^{2}\right\rangle=N_{m} / N_{s}$ where $N_{m}$ is the peak ion density and $N_{s}$ is the ion density at the satellite height, are all obtained using the IRI-2007 model. The thickness used in the calculation varies from 60 to $80 \mathrm{~km}$, and the height of the phase screen is from 300 to $500 \mathrm{~km}$, depending on the location and local time of the observation. In the simulation, we make some more assumptions on the parameters. The wavelength $(\lambda)$ of the signal is $25 \mathrm{~cm}$. The zenith angle is assumed to be 0 when the wave is the vertical incidence. The velocity of the probe $\left(V_{p}\right)$ is $7.6 \mathrm{~km} \mathrm{~s}^{-1} . Z$ is derived from the satellite height $(600 \mathrm{~km})$ and the height of the phase screen obtained from the IRI-2007 model. For the assumption of isotropic irregularity, $F$ equals 1 .

\section{DATA PROCESSING}

ROCSAT-1, a scientific satellite of the Republic of China, operated from March 1999 to June 2004 at an altitude of $600 \mathrm{~km}$ with a $35^{\circ}$ inclined orbital plane. Onboard the satellite, Ionospheric Plasma and Electrodynamics Instrument (IPEI) sampled the ion concentration with a normal mode at $32 \mathrm{~Hz}$, and a fast mode at $1024 \mathrm{~Hz}$.

Su et al. (2006) have used the 1-s averaged density data from ROSCAT-1 to calculate the distribution of irregularity occurrence rates at different local times, seasons, latitude and/or longitude, magnetic disturbance index and solar activity. Following the study of Su et al. (2006), the irregularities are identified in each 8-s segment of data by the following auto-search algorithm for the FFT processing

$\sigma=\frac{\left[\frac{1}{8} \sum_{i=1}^{8}\left(\log n_{i}-\log n_{o i}\right)^{2}\right]^{1 / 2}}{\frac{1}{8} \sum_{i=1}^{8} \log n_{o i}}$

where the $n_{i}$ is the 1-s averaged ion density and $n_{o i}$ is the linearly fitted value at the $i^{\text {th }}$ data point. $\sigma$ is the fluctua- tion character of the ion density in a 8-s segment of data stream. When $\sigma$ is larger than a pre-defined threshold such as greater or equal to 0.3 , for example, it will be regarded as an irregularity structure. Thereafter, we use FFT to perform a spectral analysis for the identified segment with the original 32 sampled data points. First, the data segment is linearly detrended and the Hanning window is added for energy leakage in the spectrum.

In the following data process, we have modified the procedure used by Wernik et al. (2007) by substituting the $C_{s}$ value in Eq. (1) with Eq. (2). Then $P_{s}\left(f_{i}\right)$ becomes a function of electron variance $\left\langle\Delta N_{e}^{2}\right\rangle$, the outer scale $\left(\lambda_{0}\right)$ and the spectral index ( $p)$, as seen in Eq. (6),

$$
P_{s}\left(f_{i}\right)=\frac{2\left\langle\Delta N_{e}^{2}\right\rangle q_{0}^{p-1} \Gamma\left(\frac{p}{2}\right) f_{i}^{-p}}{\sqrt{\pi} V_{p} \Gamma\left(\frac{p}{2}-\frac{1}{2}\right)\left[\left(\frac{2 \pi}{\lambda_{0} f_{i}}\right)^{2}+\left(\frac{2 \pi}{V_{p}}\right)^{2}\right]^{p / 2}}
$$

In fitting Eq. (6), we treat $\left\langle\Delta N_{e}^{2}\right\rangle, p$ and $\lambda_{0}$ as variables to be obtained from the fitting process. However, the $\left\langle\Delta N_{e}^{2}\right\rangle$ obtained directly from the variance of the spectrum is slightly different from the fitted density variance, $\left\langle\Delta N_{e}^{2}\right\rangle^{*}$, obtained from the curve fitting of Eq. (6). The reason is that there exists a normalization factor between the powers in the frequency and time domain.

The trust region method is one of the algorithms in the optimization theory which provides the best solution under pre-defined conditions. In the algorithm, there are several adjusted parameters such as size of the region, moving direction of fitting and convergence conditions to obtain the desired variables during each iteration of the curve fitting procedure. We can have a clear picture of the trust region method from the flow chart shown in Fig. 1 (Thomas and Li 1996; Sun and Yuan 2006). In our case, we use Eq. (6) to fit the observed spectrum of the density measurement, $y\left(f_{i}\right)$, in the least-squares fitting process to obtain three variables, $\lambda_{0},\left\langle\Delta N_{e}^{2}\right\rangle^{*}$ and $p$. The parameter $f_{i}$ is determined by the sampling rate of density measurement, and the initial trust regions for the three variables are as follows: an outer scale $\lambda_{0}$ between $1 \sim 500 \mathrm{~km}$, spectral index $p$ between $1 \sim 4$ and $\log _{10}\left(\left\langle\Delta N_{e}^{2}\right\rangle^{*}\right)$ between the measured value in the 8-s data segment of $\log _{10}\left(\left\langle\Delta N_{e}^{2}\right\rangle^{*}\right) \pm 2$.

Equation (6) is fitted in the log-log scale of SDF for the ion density within the frequency $7.6 \mathrm{~Hz}$ to avoid noise contamination in the high frequency range. The procedure is illustrated in Fig. 2. The top panel shows the 8-s data segment in time series with a linear trend added, the second panel is the detrended data with the Hanning window, and the bottom panel shows the fitted curve to the spectrum.

After the curve fitting, we obtain the turbulence strength parameter to calculate the scintillation index $\mathrm{S}_{4}$ from Eqs. (2) and (3). Wernik et al. (2007), on the other hand, used the spectrum of the ionospheric electron density from the 


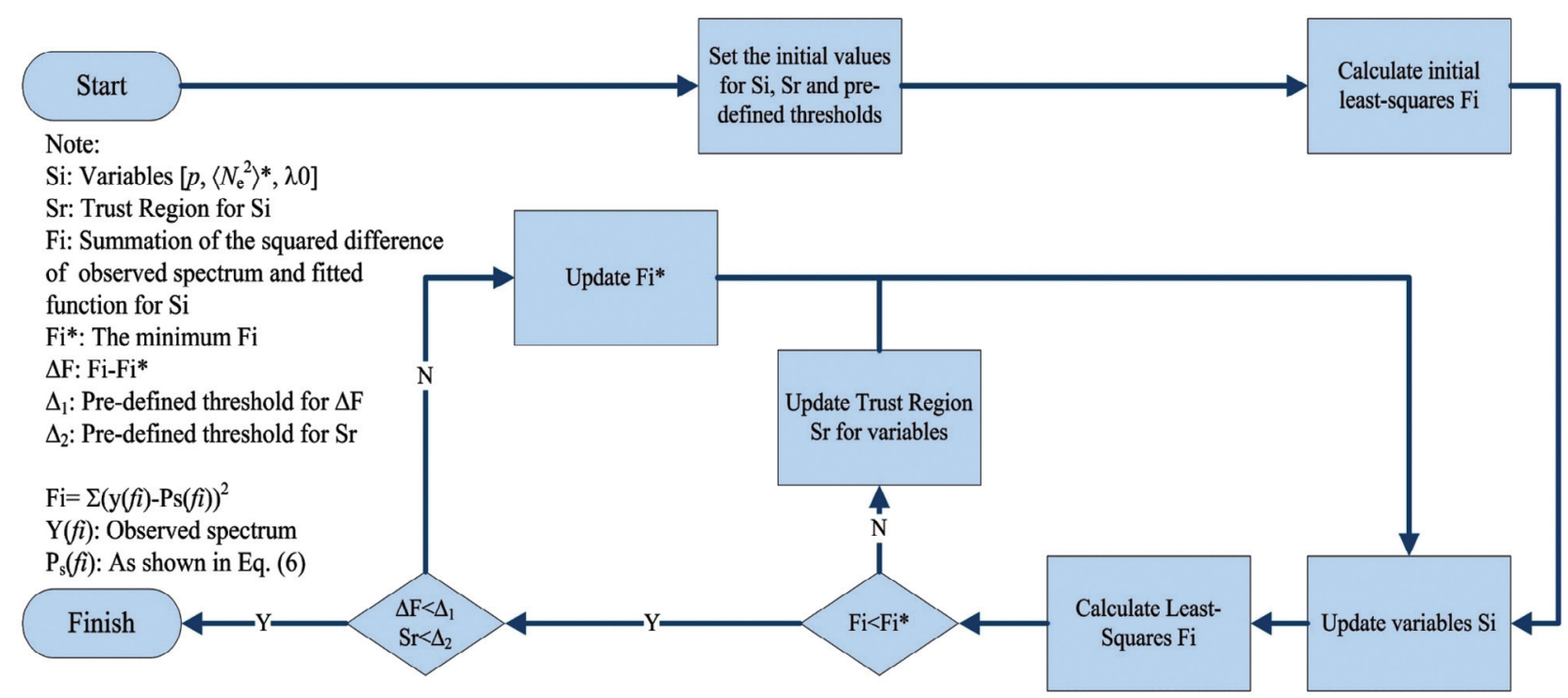

Fig. 1. The flow chart for the trust region method.
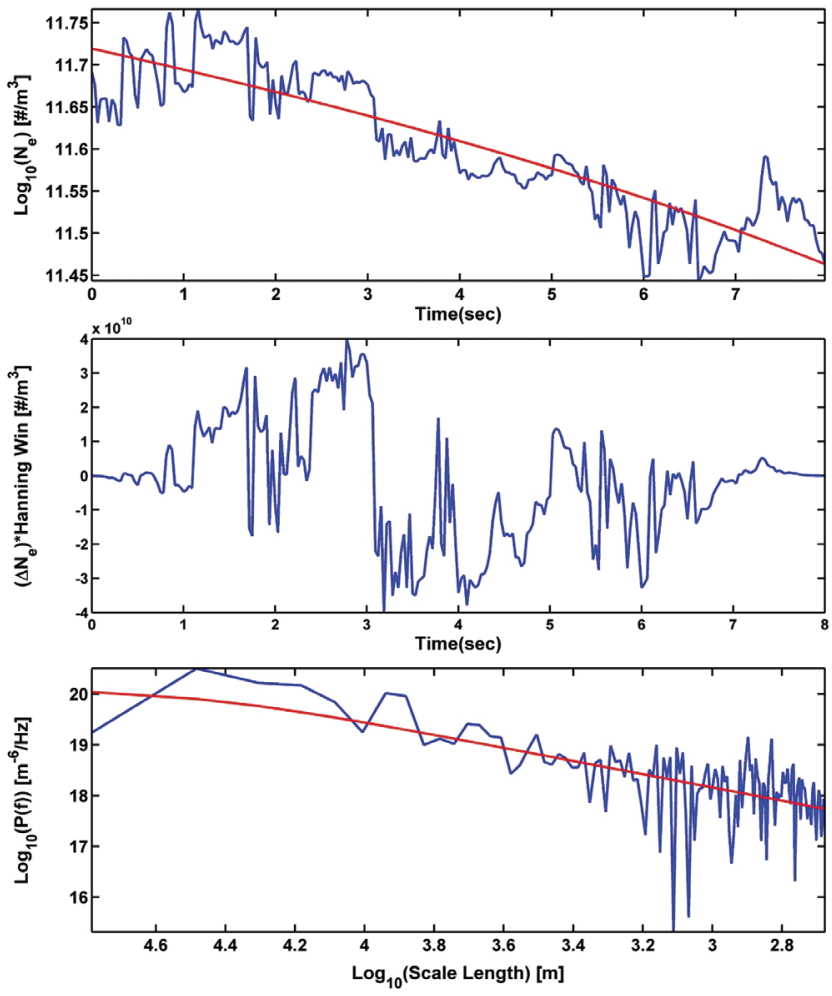

Fig. 2. Example of ion density measurement from ROCSAT-1 and the sample of data processing procedure with the fitted parameters, $p=$ $1.31, \lambda_{0}=30.6 \mathrm{~km}$ and $\left\langle\Delta N_{e}^{2}\right\rangle^{*}=7.69 \times 10^{20} \mathrm{~m}^{-6}$.

Dynamics Explore 2 (DE2) in the frequency band of 1 $20 \mathrm{~Hz}$ to derive the spectral index under the condition of $2 \pi f / V_{p} \gg q_{0}$. The turbulent parameter $C_{s}$ is then estimated from Eq. (1) at several chosen frequencies from the fitted spectrum. However, it is noted that from the outer scale dis- tribution in Fig. 3 obtained in the current study, some outer scales will violate the condition, $2 \pi f / V_{p} \gg q_{0}$.

\section{RESULTS AND DISCUSSION}

\subsection{Distributions of the Spectral Index}

The spectral index of density variation is an important parameter indicating the power distribution of ionospheric density turbulence over different scales. Kil and Heelis (1998) used the AE-E satellite measurement to get the spectral index to show the different types of spectrum between the irregularity at low and high altitudes. The energy cascading process of the irregularity structure can depend on the local time. According to Eqs. (1), (2), and (3), it is noted that the spectral index does play a vital role in determining the scintillation index.

Figures $3 \mathrm{a}$ and $\mathrm{b}$ show the distributions of spectral index for dip latitude of $\leq 20^{\circ}$ and $>20^{\circ}$, respectively. The results are obtained for all scintillation indices $S_{4} \leq 0.6$ during period from 2000 to 2003 . The variation of the spectral index as a function of dip latitude is shown in Fig. 3c, and of local time, in Fig. 3d. To meet the assumption of weak scintillation in the phase screen model, we use the data of $\mathrm{S}_{4} \leq 0.6$ in our analysis. The distributions of the spectral index in Figs. 3a and b appear as a normal distribution but skew to smaller values. The distribution peaks at about 1.6 $\sim 1.8$ for dip latitude $\leq 20^{\circ}$, but increases to $1.8 \sim 2.0$ for dip latitude $>20^{\circ}$. While in Wernik et al. (2003), the rocket measurements show a considerable scatter of spectral indices with an average value of $\mathrm{p}=1.7$ at frequencies below $60 \mathrm{~Hz}$. The variation of spectral index with the dip latitude (Fig. 3c) is symmetrical with respect to the dip equator and increases with the dip latitude. The variation of the spectral 
index with the local time (Fig. 3d) indicates that the spectral index decreases before $2000 \mathrm{LT}$, then increases after 2100 LT.

\subsection{Distributions of the Outer Scale}

The outer scale reflects the largest scale in the irregularity structure that drives scintillation. Several earlier studies have considered a value of 5 - $20 \mathrm{~km}$ for the outer scale. For example, Basu et al. (1976) assumed the outer scale to be $20 \mathrm{~km}$ in their investigation; whereas, Yeh and Liu (1977) took the value of $10 \mathrm{~km}$ for the outer scale. Rino and Owen (1984) reported that a realistic number for the outer scale of ionospheric irregularities is effectively $10-20 \mathrm{~km}$. Wheelon (2001) has indicated that a value of 5 to $20 \mathrm{~km}$ can be assumed for the outer scale. Further, Kelly (1989) summarized that several possible procedures that develop the equatorial irregularities are classified by the scales spanning at least six orders of magnitude in a spectrum.

Therefore, with a view to further examine the outer scale distribution of ionospheric irregularities in the low and mid latitudes, the distributions of outer scales are constructed and presented in Fig. 4. In this figure, the upper panel shows the outer scale distributions in the region for the dip latitude $\leq 20^{\circ}$, while the lower panel is for the region of dip latitude $>20^{\circ}$. The largest occurrence rate in the outer scale distribution for the dip latitude $\leq 20^{\circ}$ is smaller than $25 \mathrm{~km}$ and the occurrence distribution exhibits an exponential decay towards the larger values. According to the hypotheses presented by Kelly (1989), the scale smaller than $20 \mathrm{~km}$ is dominated by GRT instability. However for the dip latitude $>20^{\circ}$, the value of the largest occurrence rate is from 25 to $50 \mathrm{~km}$. The reason for a shift in the outer scale in the two latitudinal regions could be due to the fact that the physical mechanisms for the bubble evolution process in the low latitude and mid-latitude regions are different. It is also noted that, though not shown here, the variation of the outer scale is independent of local time and solar activity.

It should be noted that the outer scale derived from the turbulent theory shows the physical meaning of the irregularity evolving mechanism. Considering the calculation of the scintillation index, the phase screen approach is applicable under the weak scintillation which is dominated by the density fluctuation around the Fresnel scale. Because the outer scale is larger than the Fresnel scale at the frequency of our interest, the Fresnel filtering will remove any effects of the outer scale on the intensity scintillation (Rino 1979). Thus, the phase screen approach is valid regardless of the size of outer scale.

\subsection{The Seasonal/Latitudinal/Local-Time Distributions of Scintillation Occurrences}

The equatorial scintillation activity is controlled by
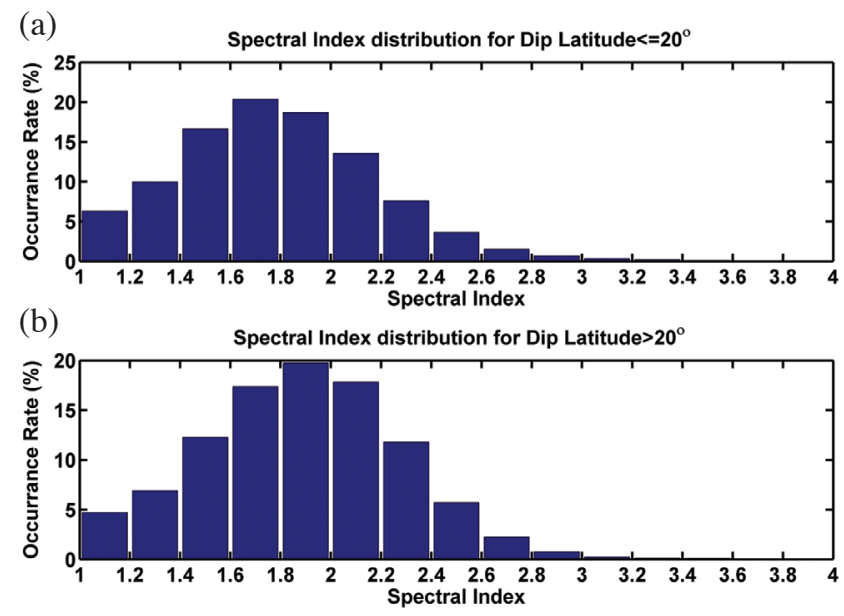

(c)

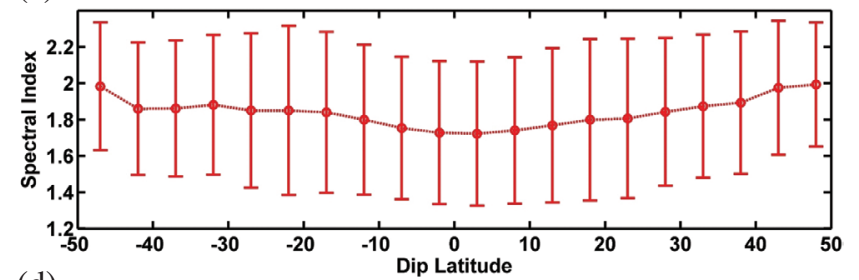

(d)

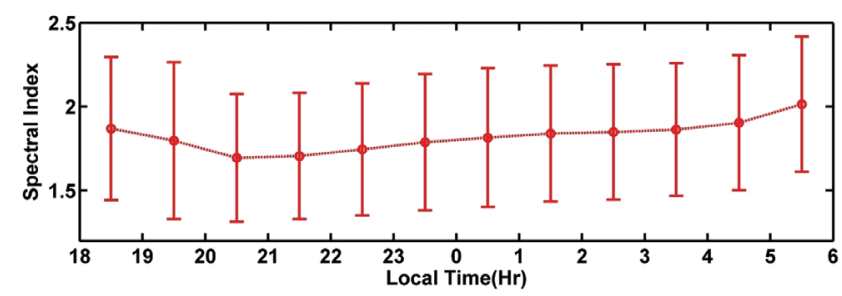

Fig. 3. (a) (b) The distributions of the spectral index obtained from curve fitting to the scintillation spectra of 2230618 -s scintillation data segments for dip latitude $\leq 20^{\circ}$ and $>20^{\circ}$ during the period from 2000 to 2003. (c) Variation of spectral index as a function of dip latitude. (d) Variation of the spectral index as a function of local time.
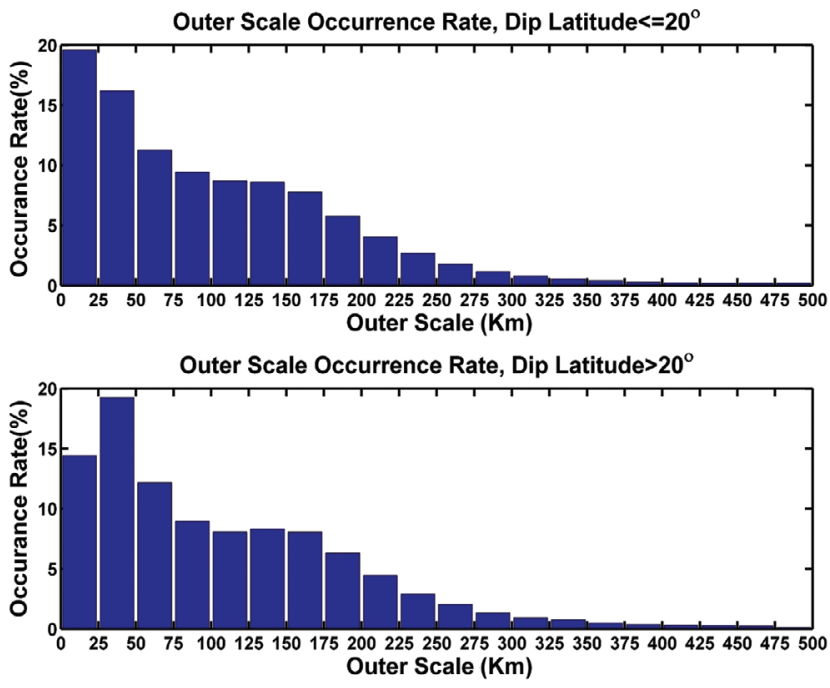

Fig. 4. Distributions of the outer scale in the regions of dip latitude $\leq$ $20^{\circ}$ and $>20^{\circ}$. 
several factors, such as local time, dip-latitude, longitude, solar activity as well as geomagnetic activity. All these factors have been studied for several years in the past. Here we present the global distribution of scintillation activity from 2000 to 2003 in local time versus the dip latitude in
Fig. 5, and separated in four seasons in Fig. 6. Since Aarons (1982) mentioned that the weak and strong scintillations can be classified as below and above the $9 \mathrm{~dB}$ level in the received signal fluctuation, respectively, we thus choose $\mathrm{S}_{4}$ $=0.3$ which is equivalent to $6 \mathrm{~dB}$ to separate the scintillation
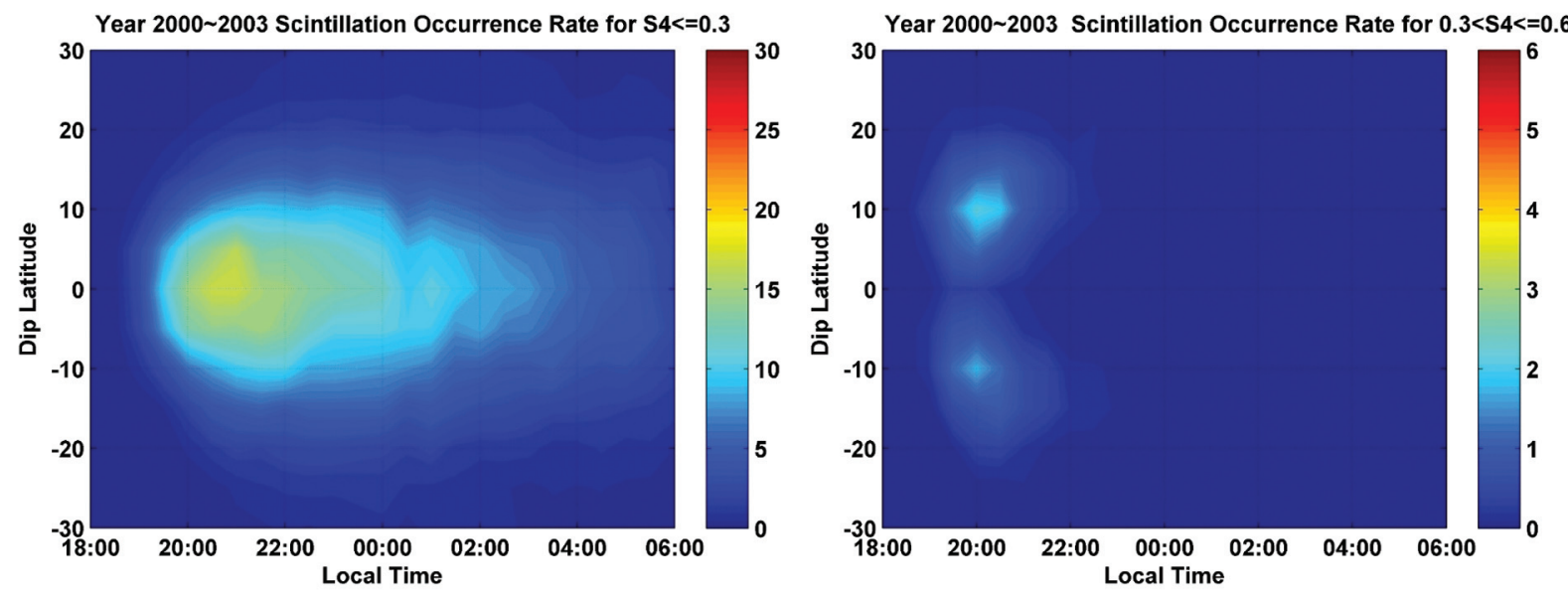

Fig. 5. The dip latitude/local-time variations of L-band scintillation occurrence rates for $\mathrm{S}_{4} \leq 0.3$ and $0.3<\mathrm{S}_{4} \leq 0.6$.
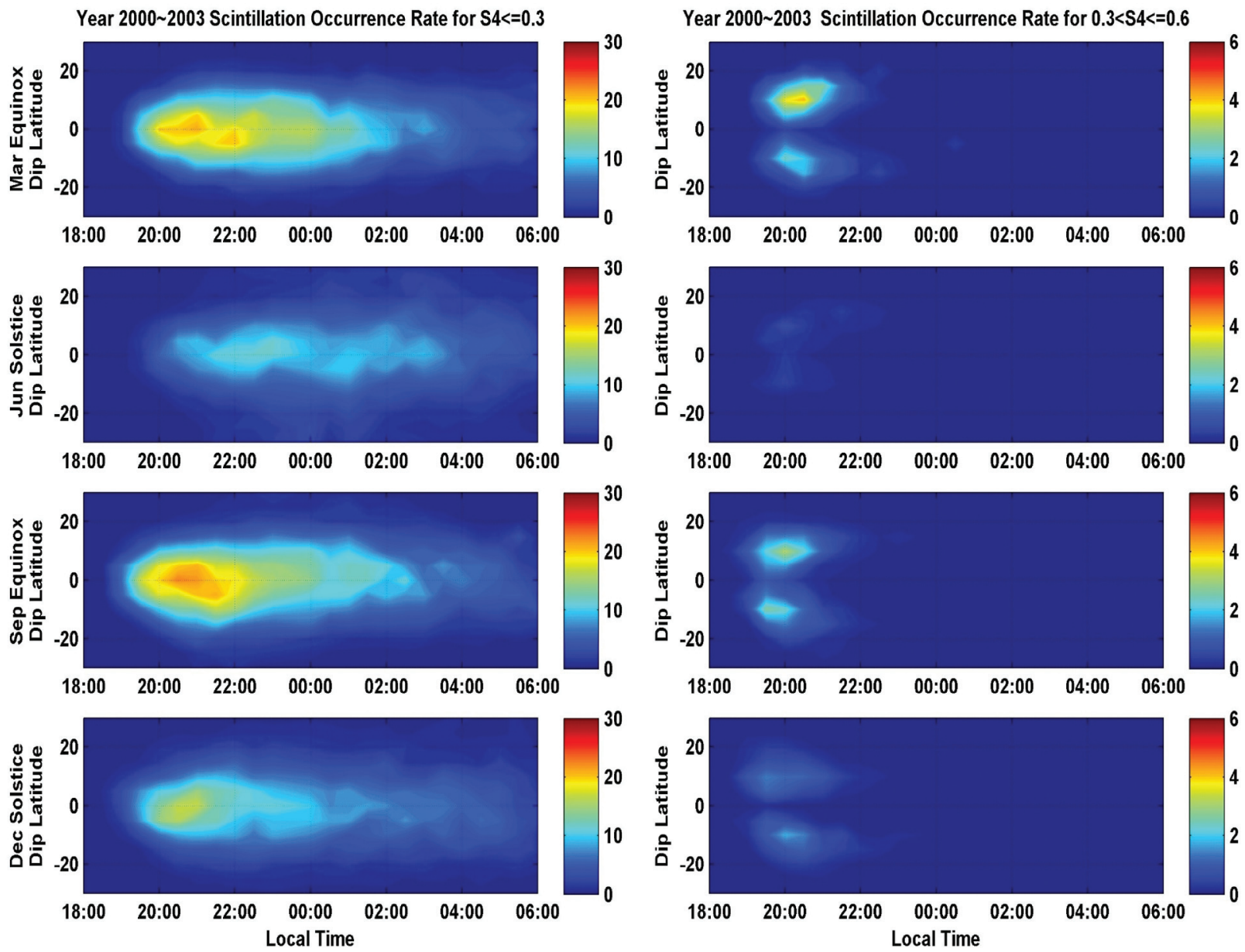

Fig. 6. The seasonal variation of the dip-latitude/local-time distributions of L-band scintillation occurrence rates for $S_{4} \leq 0.3$ and $0.3<S_{4} \leq 0.6$. 
intensity as weak or strong for the purpose of illustration. Because the close relationship between the scintillation and the ion density variance, the average value of variance for $\mathrm{S}_{4}$ $=0.3$ is found to be $2 \times 10^{22} \mathrm{~m}^{-6}$. This implies that the scintillation levels for $\mathrm{S}_{4} \leq 0.3$ and $0.3<\mathrm{S}_{4} \leq 0.6$ will be equivalent to the ion density variance, $\leq 2 \times 10^{22} \mathrm{~m}^{-6}$, and between $2 \times$ $10^{22}$ and $6.35 \times 10^{22} \mathrm{~m}^{-6}$, respectively.

The global scintillation shown in Figs. 5 and 6 are then separated into left and right panels for the cases of $\mathrm{S}_{4} \leq 0.3$ and $0.3<\mathrm{S}_{4} \leq 0.6$, respectively. It should be noted that the color bar that indicates the occurrence rate of scintillation with $0.3<\mathrm{S}_{4} \leq 0.6$ has been scaled down to one-fifth of the occurrence rate for the weak scintillation of $S_{4} \leq 0.3$. This is done to enhance of the contrast of the occurrence rate in the case of $0.3<\mathrm{S}_{4} \leq 0.6$. Even at first blush, these figures reveal several important features. First, the distributions are symmetrical with respect to the dip equator and occur within the dip latitude $\pm 20^{\circ}$. Second, the maximum occurrence rate for the case of $S_{4} \leq 0.3$ is located at the dip equator. However, as the scintillation increases, as in the case of 0.3 $<\mathrm{S}_{4} \leq 0.6$, the minimum appears at the magnetic equator and the maximum shifts to around the dip latitude $\pm 15^{\circ}$, at the equatorial ionization anomaly (EIA) crest region. Third, the occurrence rates are high in the two equinoxes, moderate during the December solstice and low during the June solstice.

For the case of $\mathrm{S}_{4} \leq 0.3$, it should be noted that the onset time of scintillations delays from the dip equator to higher dip-latitude region. Moreover, the scintillation occurrence starts from around $1900 \mathrm{LT}$ and extends up to $0200 \mathrm{LT}$ and then fades away as the local time advances. It can also be noticed that during the June solstice the occurrence rate of scintillation is lower than the other three seasons, and the onset is also later than the other three seasons.

For the case of $0.3<\mathrm{S}_{4} \leq 0.6$, the maxima of the scintillation activity is mainly located around $\pm 15^{\circ}$ dip latitude. Further, the scintillation occurrences for the case $0.3<\mathrm{S}_{4}$ $\leq 0.6$ also start at 1900 LT but fade away earlier around 2200 LT. The occurrence rate is maximum in Equinoxes and lower in June solstice. During the March equinox, the occurrence rate has an asymmetric distribution between the two hemispheres with slightly higher occurrences in the northern hemisphere than in the southern hemisphere.

Basu et al. (1988) have also shown that the occurrence of L-band scintillation is high around the dip equator during the pre-midnight hours, and the occurrence of intense scintillations $\left(0.3<\mathrm{S}_{4} \leq 0.6\right)$ maximizes around $\pm 15^{\circ}$ dip latitudes because of the highest levels of ambient electron densities at the EIA crest regions. Hence, the results presented here are consistent with the earlier reports by Basu et al. (1988).

\subsection{The Seasonal/Latitudinal/Longitudinal Distribu- tions of Scintillation Occurrences}

Figure 7 illustrates the latitudinal/longitudinal distributions of scintillation occurrence rates for the cases of $\mathrm{S}_{4} \leq$ 0.3 (left panels) and $0.3<\mathrm{S}_{4} \leq 0.6$ (right panels), and Fig. 8 shows the seasonal variation of the latitudinal/longitudinal distributions. Here, the occurrence rates are computed for each $5^{\circ} \times 5^{\circ}$ latitude and longitudinal bins. In Fig. 7, the maximum occurrence rate is around $0^{\circ}$ longitude for $\mathrm{S}_{4} \leq 0.3$, and the peak occurrence rate shifts to the EIA crest regions for $0.3<\mathrm{S}_{4} \leq 0.6$. during the seasonal variation displayed in Fig. 8, the maximum occurrence rate during the March equinox takes place around $0^{\circ}$ longitude for the case of $\mathrm{S}_{4}$ $\leq 0.3$. During the June solstice and September equinox, the region of maximum occurrence rate is from $0^{\circ}$ to $60^{\circ}$ longitude, while during the December solstice, the maximum occurrence rates are located from $-60^{\circ}$ to $0^{\circ}$ longitude. Basu et al. (1976) have also reported that a higher occurrence rate of the VHF scintillation ranges from $-60^{\circ}$ to $0^{\circ}$ in longitude in the winter period from the OGO-6 measurements in the year 1969 to 1970 . In $\mathrm{Su}$ et al. (2006), the variation
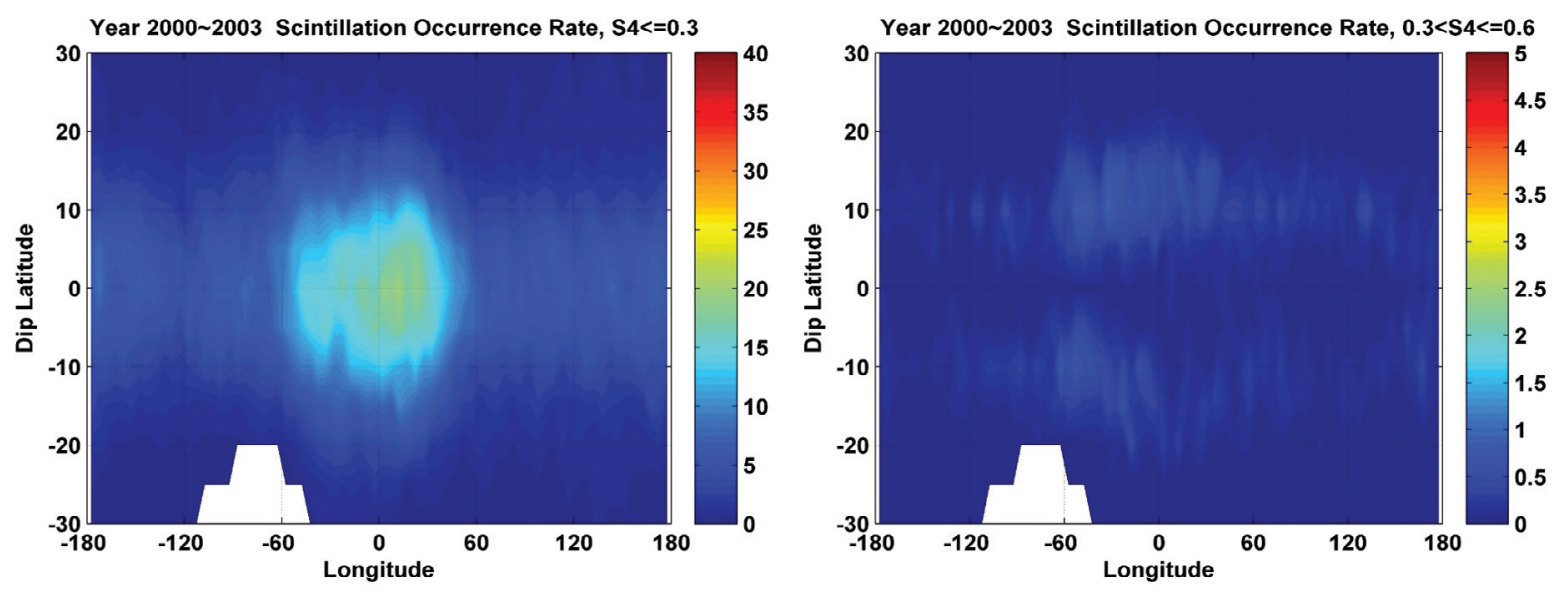

Fig. 7. The dip-latitude/longitude variations of L-band scintillation occurrence rates for $S_{4} \leq 0.3$ and $0.3<S_{4} \leq 0.6$. 

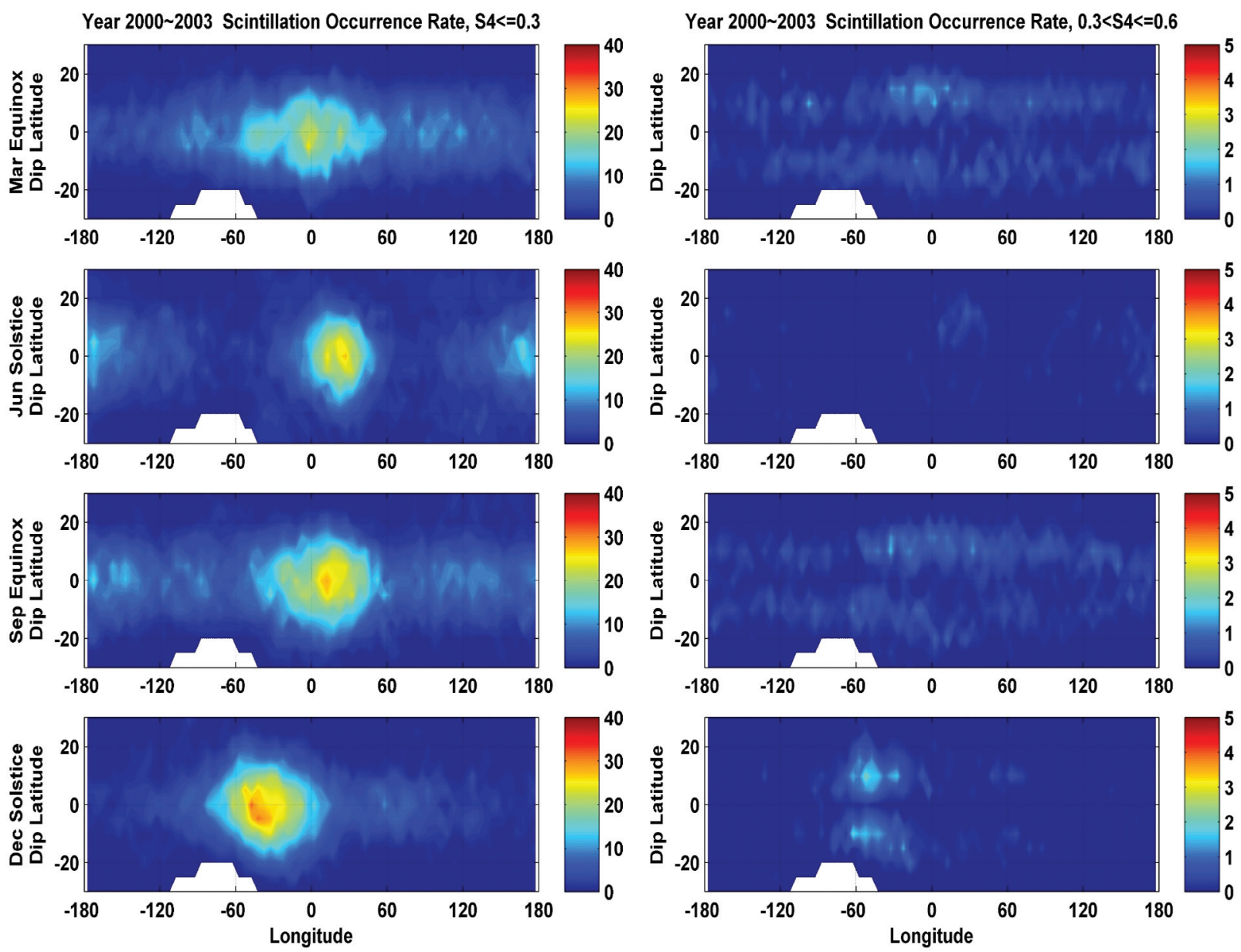

Fig. 8. The seasonal variation of the dip-latitude/longitude distributions of L-band scintillation occurrence rates for $\mathrm{S}_{4} \leq 0.3$ and $0.3<\mathrm{S}_{4} \leq 0.6$.

of irregularity occurrence rates in longitude is concentrated from $-60^{\circ}$ to $60^{\circ}$ in longitude, and is symmetrical with respect to the dip equator. We can see a similar variation of scintillation occurrences for the case of $S_{4} \leq 0.3$ in Fig. 8 . The reason for shifting the occurrence rate distribution in longitude in different seasons should come from the alignment of sunset terminator and magnetic meridian as was proposed in a model by Tsunoda (1985). The scintillation maximizes at the time of the year when the solar terminator is almost aligned with the magnetic flux tube. Even for the case of $0.3<\mathrm{S}_{4} \leq 0.6$, the occurrence rate peaks at the EIA crest regions, the seasonal shift for the region of maximum occurrence is still similar to the case of $\mathrm{S}_{4} \leq 0.3$.

\subsection{Dependence on Solar Activity}

The annual mean values of the $\mathrm{F}_{10.7}$ solar flux indices for the years 2000, 2001, 2002 and 2003 are 180, 181, 180 and 129 , respectively. Here we average the data for the years from 2000 to 2002 to plot the occurrence rate distribution for the high solar activity years, and consider the year 2003 as the lowest for solar activity. The upper two panels in Fig. 9 show the scintillation occurrence rates during the high solar activity, and the lower two ones are for the medium solar activity. It is obvious that the occurrence rate in the high solar activity period is much higher than that in the medium solar activity period for both cases of $S_{4} \leq 0.3$ and $0.3<\mathrm{S}_{4} \leq 0.6$. Basu and Basu (1992) stated that the density amplitude $(\Delta N / N)$ does not vary much with the solar activity but the background density in the solar maximum period is at least 10 times of that in the solar minimum period. Therefore, scintillation activities are enhanced due to the increased background ionization density during the higher solar activity period.

\subsection{Dependence on Geomagnetic Activity}

The effects of geomagnetic activity on the occurrence of scintillations have been studied using the observations in the year 2000. In this analysis, $\mathrm{Kp}=3$ is used to separate the condition of magnetic fields disturbed against quiet periods. Figures $10 \mathrm{a}$ and $\mathrm{b}$ show the variation of scintillation occurrence rates for quiet and disturbed periods, respectively. Comparing Fig. 10a against Fig. 10b, it is seen that for the 
case of $\mathrm{S}_{4} \leq 0.3$, the occurrence rate during quiet periods is higher and concentrated in some local regions (from longitude $60^{\circ}$ to $-60^{\circ}$ ) than that during the disturbed periods. The location with the high occurrence rate is almost the same in every season. However, during the winter solstice, under magnetically disturbed times the location of high occurrence rate shifts close to longitude $-120^{\circ}$, and the lower occurrence rate seems to scatter in longitude.

For the case of $0.3<\mathrm{S}_{4} \leq 0.6$, the high occurrence rate appears to occur along the EIA region in the magnetically quiet time. However, the high occurrence rate seems scattered within the dip latitude $< \pm 20^{\circ}$ in the magnetically disturbed time. Therefore, it seems that the scintillation occurrence rate will be higher during the magnetically disturbed times than that during the magnetically quiet times at the location of longitude $20^{\circ}$ and dip latitude $0^{\circ}$ in the figure of the March equinox. Moreover, it seems that the magnetic activity plays a role in decreasing the scintillation occurrence rate at the location of longitude $0^{\circ}$ and dip latitude $18^{\circ}$ during the magnetically disturbed period (Fig. 10b).

Figure 11 shows the variation of scintillation occurrence rate in latitude and local time under different magnetic conditions. The upper two panels show the scintillation occurrence under the magnetically quiet time, and in the lower two panels, under the disturbed time. Comparing the cases of magnetic activities, we can observe more scattering and smaller occurrence rate distributed in some latitude after midnight in the case of $\mathrm{Kp} \geq 3$.

\section{CONCLUSION}

The in-situ satellite measurements of ion density provide a great database for scintillation study with its global coverage and high sampling rate. The work presented here is to show the scintillation climatological model from the density measurement around the equatorial and low latitude regions during the years 2000 to 2003 when the solar activity is high. There exist some limitations in this model as mentioned in Wernik et al. (2007), such as the peak density, the peak height, and the irregularity layer thicknesses are not observed. Furthermore, the assumption of isotropic irregularity is used in the phase screen theory.

The scintillation level derived by the curve-fitting method is similar to that of Wernik et al. (2007) except that the work of Wernik et al. is for the high latitude region. Therefore, the current report can complement Wernik et al. in the latitudinal distribution. It should also be noted that the two studies are conducted under different solar activity
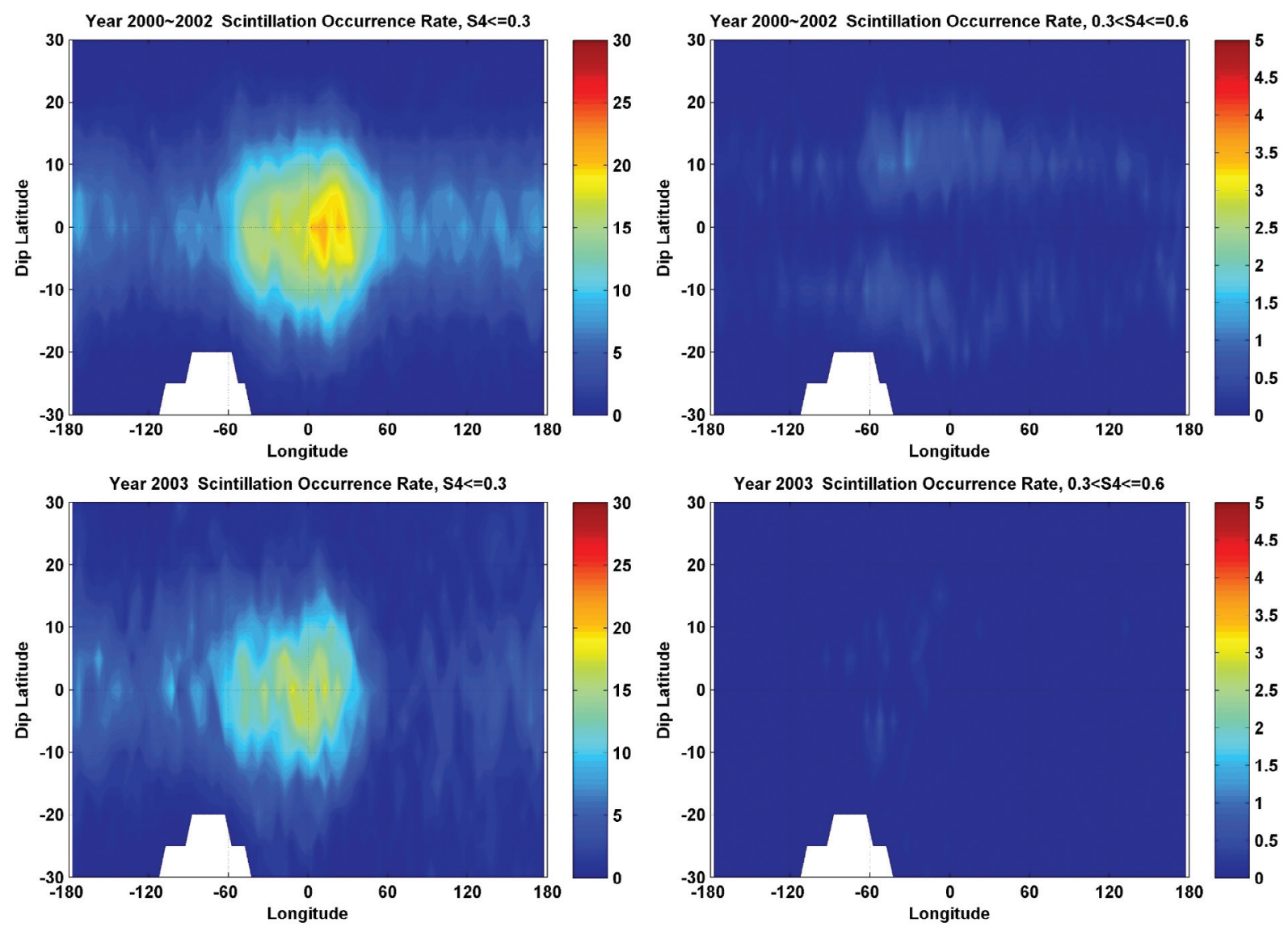

Fig. 9. The distributions of L-band scintillation occurrence rates under different solar activities. 
(a)
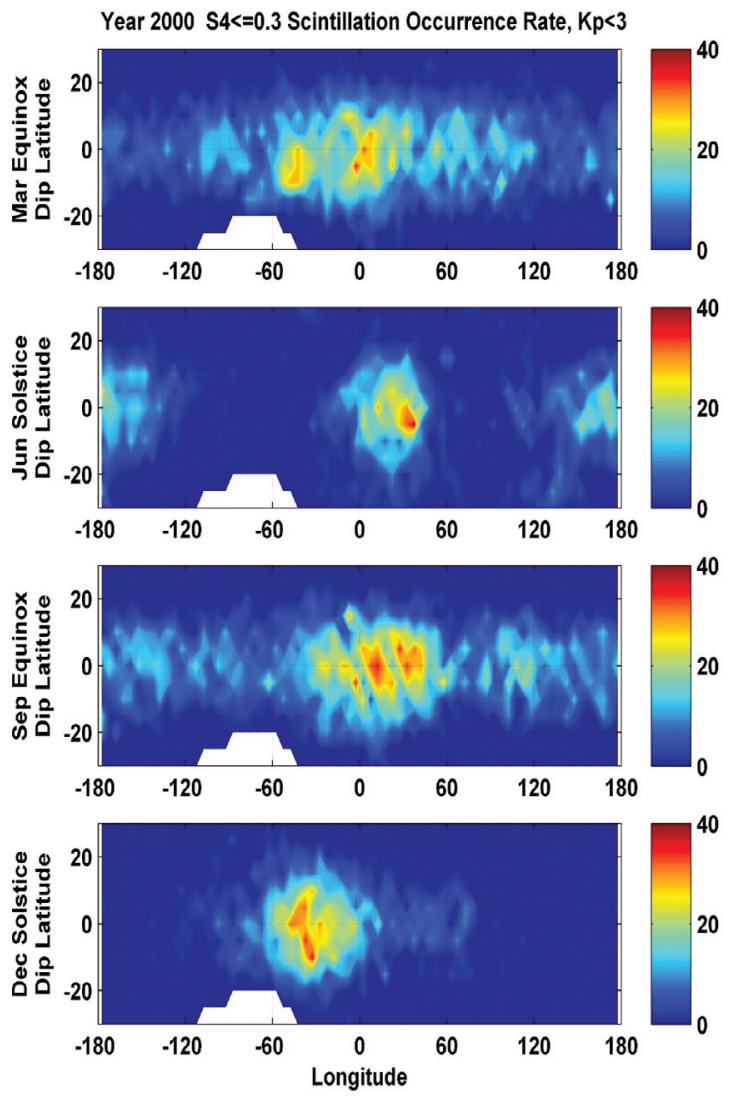

(b)
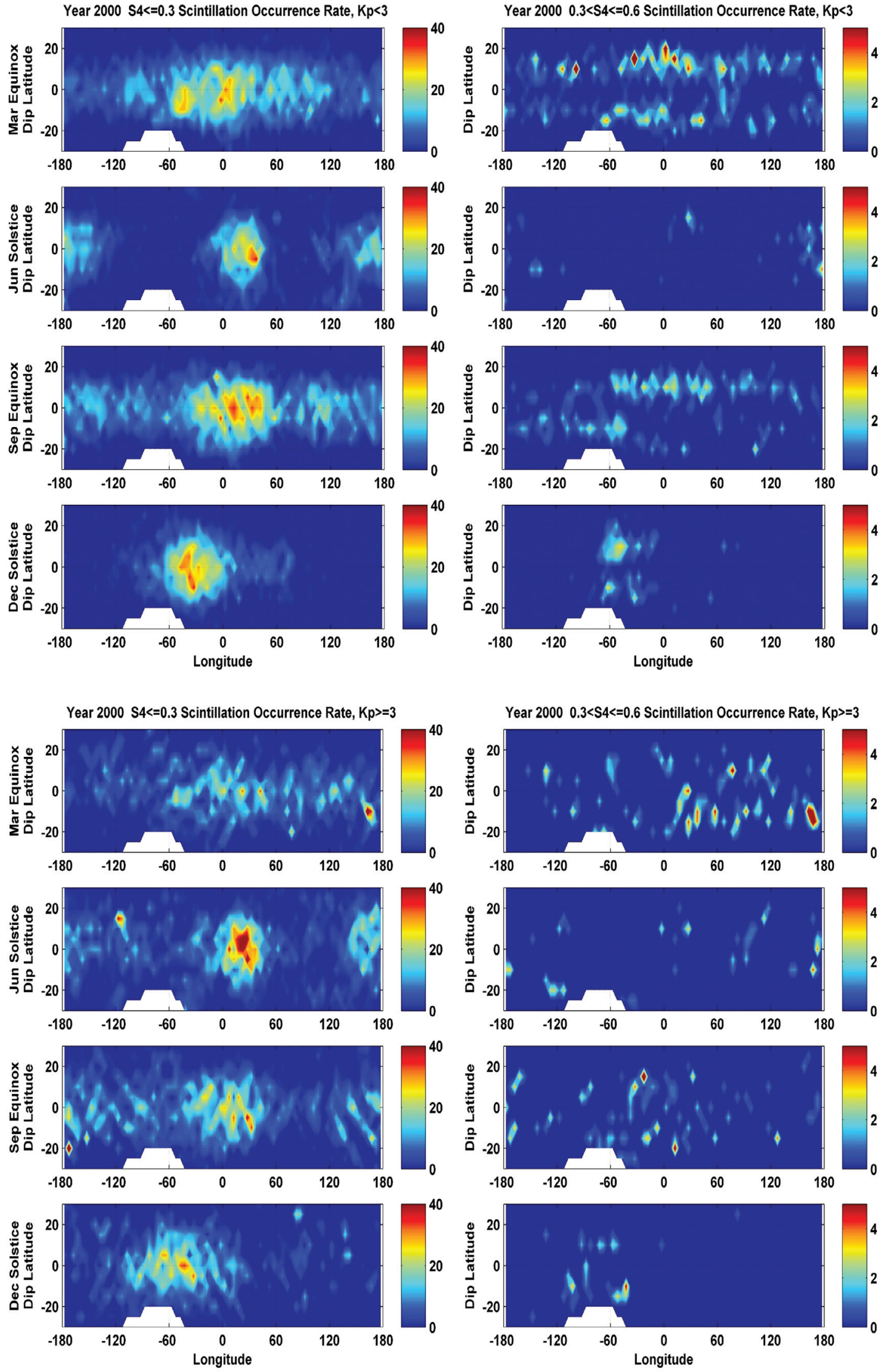

Fig. 10. (a) The seasonal variation of the dip-latitude/longitude L-band scintillation occurrence rates of $\mathrm{S}_{4} \leq 0.3$ and $0.3<\mathrm{S}_{4} \leq 0.6$ under magnetically quiet times. (b) The seasonal variation of the dip-latitude/longitude L-band scintillation occurrence rate of $\mathrm{S}_{4} \leq 0.3$ and $0.3<\mathrm{S}_{4} \leq 0.6$ under magnetically disturbed times. 

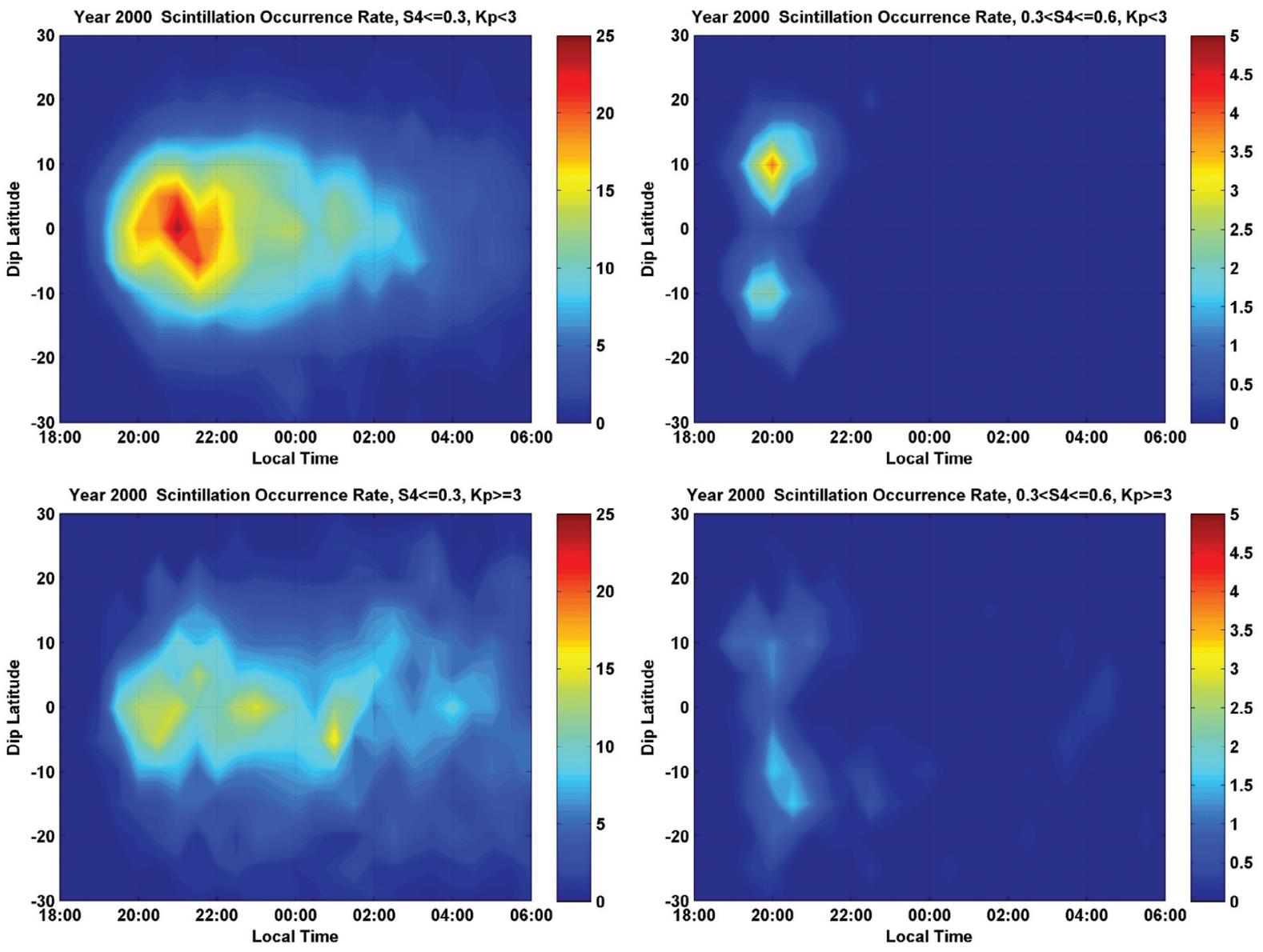

Fig. 11. The dip-latitude/local-time L-band scintillation occurrence rates of $S_{4} \leq 0.3$ and $0.3<S_{4} \leq 0.6$ under different geomagnetic activities.

periods. Nevertheless, the two scintillation models could be incorporated to study the effect of scintillation for the system designer to study the spaced-borne and ground technological system with the propagation channel through the ionosphere.

In the past, the value of an outer scale used in the study of scintillation is almost a purely theoretical consideration. The distribution of an outer scale obtained in this paper is from in-situ measurements of density irregularities. Thus it should be valuable for future study of scintillation occurrences.

In the future, the relationship among the outer scale, $\mathrm{S}_{4}$, spectral index, $\Delta N_{e} / N_{e}\left(\right.$ or $\Delta N_{e}$ ) should be studied.

Acknowledgements The work is completed under a partial support of AOARD 09-4029 and 10-4104.

\section{REFERENCES}

Aarons, J., 1982: Global morphology of ionospheric scintillations. Proc. IEEE, 70, 360-378.

Aarons, J., 1993: The longitudinal morphology of equatorial F-layer irregularities relevant to their occurrence.
Space Sci. Res., 63, 209-243, doi: 10.1007/BF007507 69. [Link]

Basu, S. and S. Basu, 1985: Equatorial scintillations : Advances since ISEA-6. J. Atmos. Terr. Phys., 47, 753768, doi: 10.1016/0021-9169(85)90052-2. [Link]

Basu, S. and S. Basu, 1992: Ionospheric structure and scintillation. In: Tatarskii, V. I., A. Ishimaru, V. U. Zavorotny, etc. (Eds.), Wave Propagation in Random Media (Scintillation), 139-155, Spie Press, Bellingham Washington.

Basu, S., S. Basu, and B. K. Khan, 1976: Model of equatorial scintillations from In-situ measurements. Radio Sci., 11, 821-832, doi: 10.1029/RS011i010p00821. [Link]

Basu, S., E. MacKenzie, and S. Basu, 1988: Ionospheric constraints on VHF/UHF communications links during solar maximum and minimum periods. Radio Sci., 23, 363-378, doi: 10.1029/RS023i003p00363. [Link]

Bhattacharyya, A., K. C. Yeh, and S. J. Franke, 1992: Deducing turbulence parameters from transionospheric scintillation measurements. Space Sci. Rev., 61, 335386, doi: 10.1007/BF00222311. [Link]

Cervera, M. A., R. M. Thomas, K. M. Groves, A. G. Ram- 
li, and Effendy, 2001: Validation of WBMOD in the Southeast Asian region. Radio Sci., 36, 1559-1572, doi: 10.1029/2000RS002520. [Link]

Kil, H. and R. A. Heelis, 1998: Equatorial density irregularity structures at intermediate scales and their temporal evolution. J. Geophys. Res., 103, 3969-3981, doi: 10.1029/97JA03344. [Link]

Li, S. Y. and C. H. Liu, 2004: Modeling the effects of ionospheric scintillation on LEO satellite communication. IEEE Commun. Lett., 8, 147-149.

Rino, C. L., 1979: A power law phase screen model for ionospheric scintillation 1. Weak scatter. Radio Sci., 14, 1135-1145, doi: 10.1029/RS014i006p01135. [Link]

Rino, C. L. and E. J. Fremouw, 1977: The angle dependence of singly scattered wavefields. J. Atmos. Terr. Phys., 39, 859-868, doi: 10.1016/0021-9169(77)9016 6-0. [Link]

Rino, C. L. and J. Owen, 1984: Numerical simulations of intensity scintillation using the power law phase screen model. Radio Sci., 19, 891-908, doi: 10.1029/RS019i0 03p00891. [Link]

Secan, J. A., R. M. Bussey, E. J. Fremouw, and S. Basu, 1995: An improved model of equatorial scintillation. Radio Sci., 30, 607-617, doi: 10.1029/94RS03172. [Link]

Secan,J. A., R. M. Bussey, E. J. Fremouw, and S. Basu, 1997: High-latitude upgrade to the Wideband ionospheric scintillation model. Radio Sci., 32, 1567-1574, doi: 10.1029/97RS00453. [Link]

Su, S. Y., C. H. Liu, H. H. Ho, and C. K. Chao, 2006: Dis- tribution characteristics of topside ionospheric density irregularities: Equatorial versus midlatitude regions. J. Geophys. Res., 111, A06305, doi: 10.1029/2005JA 011330. [Link]

Sun, W. and Y. X. Yuan, 2006: Optimization Theory and Methods: Nonlinear Programming, Springer, New York, 699 pp.

Thomas, F. C. and Y . Li, 1996: An interior Trust Region approach for nonlinear minimization subject to bounds. SIAM J. Optim ., 6, 418-445.

Tsunoda, R. T., 1985: Control of the seasonal and longitudinal occurrence of equatorial scintillations by the longitudinal gradient in integrated E region Pedersen conductivity. J. Geophys. Res., 90, 447-456, doi: 10.1029/ JA090iA01p00447. [Link]

Wernik, A. W., L. Alfonsi, and M. Materassi, 2007: Scintillation modeling using in situ data. Radio Sci., 42, RS1002, doi: 10.1029/2006RS003512. [Link]

Wernik, A. W., J. A. Secan, and E. J. Fremouw, 2003: Ionospheric irregularities and scintillation. Adv. Space Res., 31, 971-981, doi: 10.1016/S0273-1177(02)00795 -0 . [Link $]$

Wheelon, A. D., 2001: Electromagnetic Scintillation I, Cambridge University Press, Cambridge, UK.

Yeh, K. C. and C. H. Liu, 1977: Diagnosis of the turbulent state of ionospheric plasma by propagation methods. Radio Sci., 12, 1031-1034, doi: 10.1029/RS012i0 06p01031. [Link]

Yeh, K. C. and C. H. Liu, 1982: Radio wave scintillations in the ionosphere. Proc. IEEE, 70, 324-360. 